\title{
Numerical study of long Josephson junctions coupled to a high-Q cavity
}

\author{
Grønbech-Jensen, N.; Pedersen, Niels Falsig; Davidson, A.; Parmentier, R. D.
}

Published in:

Physical Review B

Link to article, DOI:

10.1103/PhysRevB.42.6035

Publication date:

1990

Document Version

Publisher's PDF, also known as Version of record

Link back to DTU Orbit

Citation (APA):

Grønbech-Jensen, N., Pedersen, N. F., Davidson, A., \& Parmentier, R. D. (1990). Numerical study of long Josephson junctions coupled to a high-Q cavity. Physical Review B, 42(10), 6035-6039. https://doi.org/10.1103/PhysRevB.42.6035

\section{General rights}

Copyright and moral rights for the publications made accessible in the public portal are retained by the authors and/or other copyright owners and it is a condition of accessing publications that users recognise and abide by the legal requirements associated with these rights.

- Users may download and print one copy of any publication from the public portal for the purpose of private study or research.

- You may not further distribute the material or use it for any profit-making activity or commercial gain

- You may freely distribute the URL identifying the publication in the public portal 


\title{
Numerical study of long Josephson junctions coupled to a high- $Q$ cavity
}

\author{
N. Grønbech-Jensen and N. F. Pedersen \\ Physics Laboratory I, The Technical University of Denmark, DK-2800 Lyngby, Denmark
}

A. Davidson

IBM Thomas J. Watson Research Center, Yorktown Heights, New York 10598

R. D. Parmentier

Department of Physics, University of Salerno, I-84081 Baronissi (SA), Italy

(Received 12 March 1990; revised manuscript received 30 May 1990)

\begin{abstract}
Long Josephson junctions coupled to a high- $Q$ resonator are studied numerically and compared with recently published approximative results, obtained by using a perturbative approach to the fluxon motion in the junction. The similarities and differences in the two approaches are discussed.
\end{abstract}

Recently great interest has been devoted to the subject of phase locking of soliton motion in long Josephson junctions (LJJ's). This interest is caused by the possible future use of phase-locked LJJ's as extremely narrowlinewidth microwave sources in integrated superconducting heterodyne receivers. ${ }^{1}$ In a system of phaselocked LJJ's it is possible to control the output frequency and at the same time to increase the low output power of a single junction. Experiments in the $X$ band $^{2}$ have shown that phase locking between several LJJ's is possible. $\mathrm{Nu}$ merical simulations, using the so-called particle-map approach $^{3}$ for modeling the soliton motion in the LJJ, have demonstrated phase locking between two LJJ's coupled through a linear resonator.

The purpose of the present paper is to study the phase locking of a LJJ to a cavity and to another LJJ through a cavity, by doing a full numerical simulation of the corresponding partial differential equation (PDE). This subject is in fact quite similar to the study made in Ref. 3, and a comparison of the results of the full sine-Gordon wave equation and the particle-map approach used ${ }^{3}$ can be made. As in Ref. 3, the configuration of one or more junctions connected to a linear resonator is intended as a highly simplified model of the 20-junction experimental array described by Pagano et al. ${ }^{2}$ Their device [see Fig. 1(c) of Ref. 2] consisted of a series-biased array of overlap-geometry junctions embedded near the center of a linear distributed resonator which is one halfwavelength long at the operating frequency $(\sim 10 \mathrm{GHz})$, the whole of which is in turn coupled to a microstrip transmission line to carry the generated signals to a spectrum analyzer. Limiting attention to just the halfwavelength mode of oscillation, we model such a distributed resonator as a lumped $L C$ tank, as shown schematically in Fig. 1 of Ref. 3.

As we shall see, some details are different in the two treatments, but the overall characteristic features are the same. It should here be noted that the map approach is valid only when the LJJ is relatively long, since this model considers the soliton to be a relativistic particle with no spatial extension. The PDE approach also provides more detailed information than the map about the interaction mechanism between the nonlinear soliton resonator and the linear resonator coupled to its boundary. The cost of this is, of course, a much longer computing time.

As noted above, the perturbed sine-Gordon equation (PSGE) is used to describe the dynamics of an LJJ.,

$$
\phi_{x x}-\phi_{t t}-\sin \phi=\alpha \phi_{t}-\eta,
$$

where $\phi$ is the difference in phase between the two quantum mechanical wave functions belonging to the superconductors on each side of the junction. The spatial dimension, $x$, is normalized to the Josephson penetration depth $\lambda_{J}$ and time $t$ to the inverse Josephson plasma frequency $1 / \omega_{p}$. The perturbations to the pure sine-Gordon equation [right-hand side of Eq. (1)] are given by the damping term $\alpha \phi_{t}$ corresponding to the tunneling losses and the external bias current density $\eta$.

The finite length of the LJJ enters the problem through specification of the boundary conditions at $x=0$ and $x=l$.

$$
\begin{aligned}
& \phi_{x}(0, t)=-\kappa_{0}, \\
& \phi_{x}(l, t)=\kappa_{1},
\end{aligned}
$$

where $\kappa_{0}$ and $\kappa_{1}$ are the normalized currents flowing into the LJJ through the boundaries at $x=0$ and $x=l$. The corresponding power input to the LJJ through the boundaries is then given by

$$
P=\phi_{x}(0, t) \phi_{t}(0, t)+\phi_{x}(l, t) \phi_{t}(l, t) .
$$

By connecting the boundary at $x=l$ to a parallel $L C$ tank circuit through a coupling resistance $R$ we get the normalized boundary conditions:

$$
\begin{aligned}
& \kappa_{1}=\frac{1}{R}\left[V_{0}(t)-\phi_{t}(l, t)\right], \\
& \kappa_{0}=0, \\
& V_{0, t t}=-\frac{1}{R C} V_{0, t}(t)-\frac{1}{L C} V_{0}(t)+\frac{1}{R C} \phi_{t t}(l, t) .
\end{aligned}
$$


Here $\phi_{t}(x, t)$ is the normalized instantaneous voltage across the junction, $V_{0}(t)$ the tank voltage, and $\phi_{x}(0, t)=0$ is assumed. Voltages are normalized to $\hbar \omega_{p} / 2 e$. We note here that instead of specifying $R, L, C$ we may specify the normalized cavity resonance frequency by

$$
\omega_{0}^{2}=1 /\left(L C \omega_{p}^{2}\right)-a^{2},
$$

the cavity damping constant $a=1 /\left(2 R C \omega_{p}\right)$ and the coupling resistor $R$, where $R$ below will be understood normalized to the characteristic impedance $\sqrt{\bar{L} / \bar{C}}$, where $\bar{L}$ and $\bar{C}$ are the inductance and capacitance per unit length of the LJJ.

The equations to solve for a single junction connected to the tank are then Eq. (1) and Eqs. (4). This is done by discretizing the spatial dimension and approximating the spatial derivative by a fourth-order five-point finite difference in the interior of the system and a second-order three-point finite difference near the boundaries. This combined five- and three-point approximation to the second-order spatial derivative of $\phi$ has been necessary to eliminate diverging spurious oscillations, caused by the discretization of the spatial dimension. The integration in time was performed by a standard fourth order Runge Kutta method. The spatial grid size and the time step size were varied to find appropriate convergence for the integration.

A typical behavior of the soliton motion in the system considered is shown in Fig. 1. Here a steady-state periodic interaction between the tank voltage and the Josephson junction voltage is found for a soliton frequency $\omega_{s}$ just above the tank resonance frequency $\omega_{0}$. The parameters used are $\alpha=0.05, l=4, R=7, a=\frac{1}{200}, \omega_{0}=0.75$ and $\eta=0.5$. Figure 1(a) shows the tank voltage $V_{0}(t)$ and the junction boundary voltage $\phi_{t}(l, t)$ as functions of time. Figure 1(b) shows the trajectory of the peak voltage in the junction-i.e., as a function of the position of the soliton. Clearly we see the effect of the tank on the boundary reflection; the power lost by the soliton to the tank is seen as the difference between the incoming and reflected peak voltages. Also at the free boundary at $x=0$ a small difference is found between the incoming and outcoming peak voltages. This is, however, mainly caused by the acceleration of the soliton towards its steady-state velocity. This is not to be compared directly with the inherent energy loss in a reflection on a semi-infinite lossy line,${ }^{5}$ since the inherent energy loss would result in an outgoing peak voltage smaller than the incoming. The phase space of the tank voltage is depicted in Fig. 1(c). The near harmonic motion is seen as the near elliptical trace. The "bump" indicated by an arrow shows the effect of the soliton hit on the tank. In the particle representation of the soliton, used in Ref. 3, the tank phase-space trajectory would be a displacement away from the origin followed by a damped spiral-type motion $360^{\circ}$ around the origin until the next soliton hit displaced the trajectory outwards again. The same kind of motion is found in Fig. 1(c), but clearly the soliton hit is much smoother than a sudden displacement. This is due to the fact that the soliton reflection time is not negligible compared with the period of the tank motion, as also seen from Fig. 1(a). By choosing the tank resonance frequency $\omega_{0}$ smaller, the soliton reflection time will of course be relatively smaller; hence small values of $\omega_{0}$ will increase the validity of the particle model used in Ref. 3. A decreased value of $\omega_{0}$ should of course be considered together with an increased

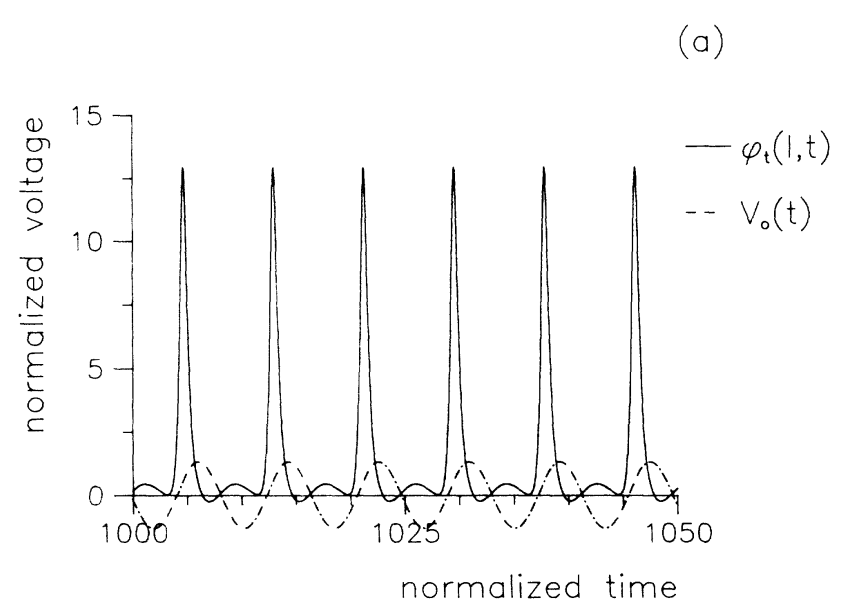

(b)

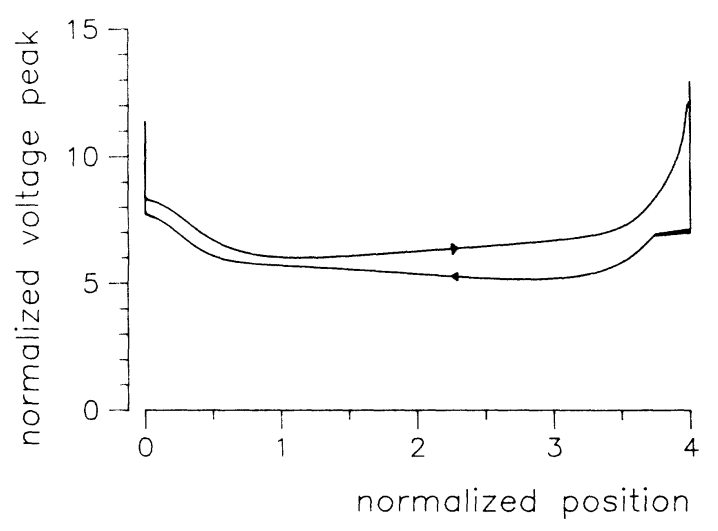

(c)

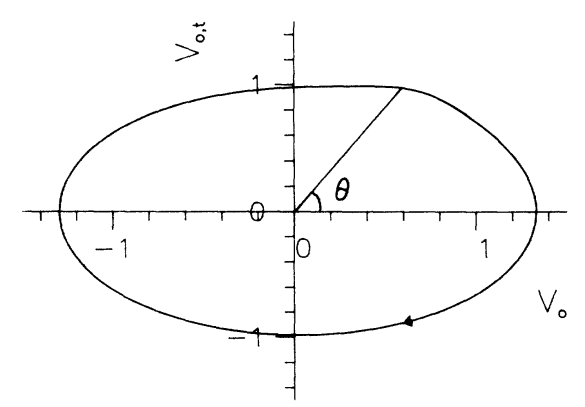

FIG. 1. The time evolution for the system of one soliton resonator coupled to a linear tank is shown for a parameter set of the following: $\alpha=0.05, l=4, \eta=0.5, R=7, a=\frac{1}{200}, \omega_{0}=0.75$. (a) Junction voltage at the tank boundary and the tank voltage vs time. (b) The voltage peak value vs its position. (c) Phase space of the tank voltage. The arrow indicates the effect of the interaction with the soliton. 
length of the junction in order to match the soliton frequency with $\omega_{0}$. A characteristic angle $\theta$ of the interaction is defined by $\theta=\Delta T \omega_{s}$ where $\Delta T$ is the time interval between the maxima of the tank voltage and the boundary voltage $\phi_{t}(l, t)$, and $\omega_{s}$ is the soliton frequency, running back and forth. The angle $\theta$ is visualized as an arrow (showing the soliton hit) in Fig. 1(c). This angle was found to vary in the interval of approximately $-\pi / 2 \rightarrow \pi / 2$ for locked states between soliton and tank motion, when the bias was varied. The angle $\theta=0$ corresponds to the soliton frequency $\omega_{s}$ at $\omega_{0}, \theta<0$ to $\omega_{s}<\omega_{0}$ and $\theta>0$ to $\omega_{s}>\omega_{0}$. Clearly the motion in Fig. 1 corresponds to the case $\theta>0$.

The tank amplitude had, of course, a maximum for $\theta=0$ and decreased as $|\theta|$ was increased. We remark here that the tank phase space motion seems to provide the best criterion for deciding when steady-state motion is obtained. Typically we had to integrate the system up to 500 time units for $a=\frac{1}{100}$ and up to 1000 time units for $a=\frac{1}{200}$ to find steady-state motion. In particular for motion with $\omega_{s}<\omega_{0}$ (low bias) we found that the transient time increased drastically.

Apart from the finite width of the soliton there are other differences between the treatment in this paper and the one made in Ref. 3. While the mapping in the particle model gives the possibility of using extremely long integration times, it loses every information of what is happening between the soliton tank collisions. In the PDE approach the long integration times are very inconvenient, but all interactions between the soliton tank reflections are retained. An obvious difference between the map approach and the full simulation of Eq. (1) and Eqs. (4) is the delta function approximation of the boundary voltage. When low bias motion in a relatively short junction (high $\omega_{0}$ ) is studied, the approximation is especially bad, but also for large bias values can there be a difference, even if the reflection time is much smaller than the tank motion period. For high bias values the plasma oscillations generated at the tank boundary during a reflection are amplified, since the tank voltage is positive for a large time after the reflection (because of the angle $\theta>0$ ); i.e., power is flowing from the tank into the junction. This is important to the stability of the soliton motion, since states with high bias easily switch to a nonlocalized rotating state. Typically we were not able to find soliton motion in the $l=4, \alpha=0.05$ junction for bias values larger than 0.8 with coupling resistance $R=7$. Also for soliton frequencies just above the tank resonance frequency $\omega_{0}$ the junction very easily switched to a nonlocalized rotating state when the bias current was changed. This may be caused by a bistability corresponding to the reported hysteresis near the tank resonance. ${ }^{3}$

In Fig. 2(a) we show a current-voltage $(I V)$ curve for the parameters noted above as well as the curve corresponding to the case without the $L C$ tank (only the shunt resistance $R$ present). The corresponding characteristic angle $\theta$ between the boundary voltage $\phi_{t}(l, t)$ and the tank voltage is shown as Fig. 2(c). From the $I V$ curve it is clear that far below the tank resonance (low bias, $\theta<0$ ) the soliton motion does not feel the $L C$ circuit, but only the resistor $R$. Increasing the bias we observe a frequency pulling towards resonant states near $\omega_{0},(\theta=0)$. Increasing the bias further we observe that the $I V$-curve approaches a new state without interaction with the $L C$ tank and $\theta$ approaches $\pi / 2$. In Fig. 2(a) the currentvoltage curve is also shown for the system without the $L C$ tank-i.e., $R=7, R C \rightarrow \infty, L C \rightarrow \infty \quad\left(V_{0}(t)=0\right)$. The fact that the interaction through $R$ is small when $\omega_{s}$ is very different from $\omega_{0}$ is also seen from Fig. 2(b), where the tank voltage amplitude is shown. From this it is found that the stored energy increases drastically near the resonance. Also the characteristic phase angle $\theta$ between the soliton motion and the tank motion, Fig. 2(c), indicates how the interaction is affected by the difference between $\omega_{s}$ and $\omega_{0}$. At resonance, the junction boundary voltage and the tank voltage have maxima at approximately the same times $(\theta \approx 0)$. Away from resonance, the two oscillators move approximately $\pm 90^{\circ}$ out of phase. We note that for the present parameters very small hys-

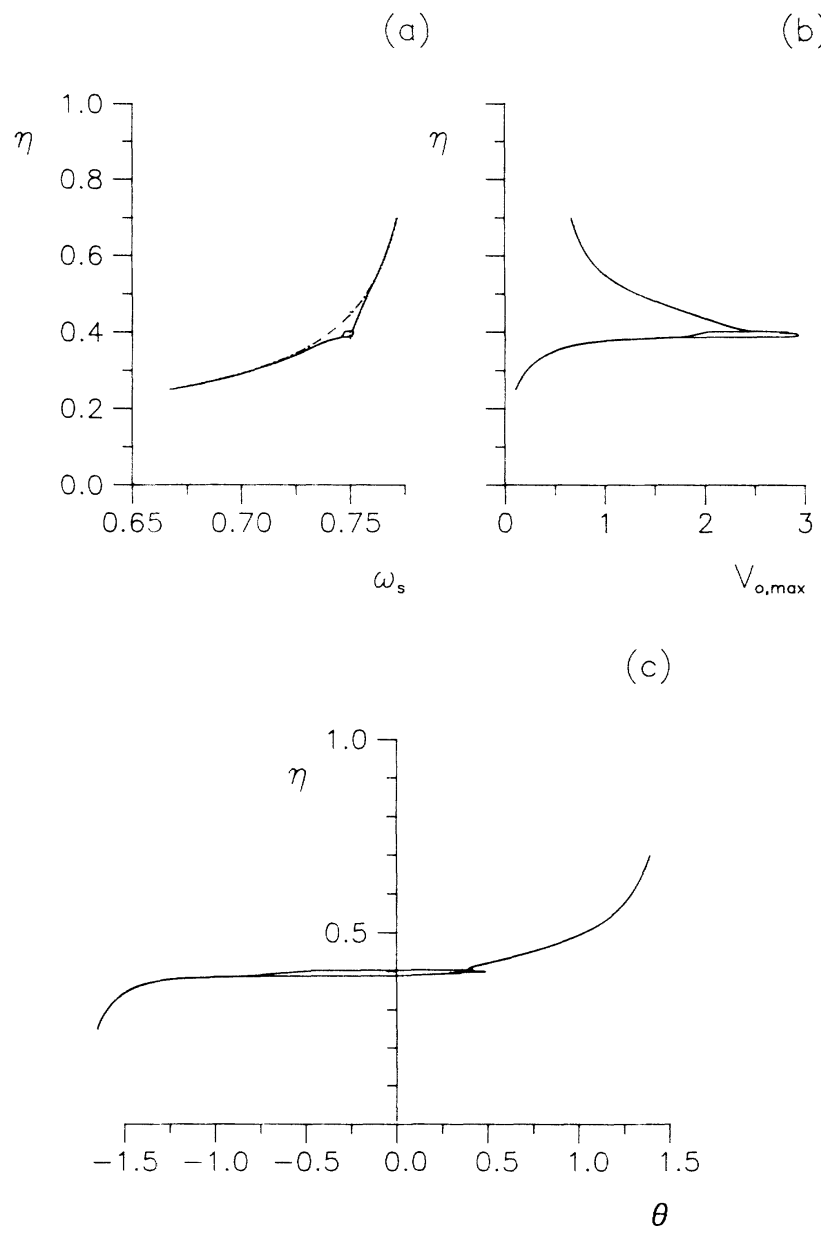

FIG. 2. Variation with the bias $(\eta)$. Parameters are as for Fig. 1. (a) Soliton frequency $\omega_{s}$ (solid curve). The soliton frequency without the LC tank (dash dotted). The vertical dotted line indicates the tank resonance frequency $\omega_{0}$. In normalized units the average voltages are $2 \omega_{\mathrm{s}}$. (b) tank voltage amplitude $V_{0, \max }$. (c) The phase difference between the soliton motion and the tank motion. 
teresis in the $I V$ curve is observed. Decreasing the damping constant $a$ slightly from the present values would create larger hysteresis near the frequency pulling region in Fig. 2(a).

The correspondence with the results of the particle model $^{3}$ can also be seen from Fig. 2(a). Some difference is observed but the essential behavior is present in both calculations. Here it should be noted that in the map approach the phaseshift of the soliton due to the boundary reflections ${ }^{6}$ was not considered. For the energy loss during the reflection at the tank the perturbation result ${ }^{7}$ obtained for $R \gg 1$ was used. Thus clearly some deviation between the map and the PDE is to be expected.

Finally we show the result of numerical experiments with two LJJ's coupled to the same resonant circuit. Apart from the structure of the coupling circuit this is similar to the situation described in Ref. 8 and is a first step in the direction of modeling the 20-junction array of Pagano et $a .^{2}$ We denote the phase differences of the first and second LJJ by $\phi$ and $\psi$, respectively. The relevant normalized equations to solve are then

$$
\begin{aligned}
& \phi_{x x}-\phi_{t t}-\sin \phi=\alpha_{1} \phi_{t}-\eta_{1}, \\
& \phi_{x}(0, t)=0, \quad \phi_{x}\left(l_{1}, t\right)=R_{12}^{-1}\left[V_{0}(t)-\phi_{t}\left(l_{1}, t\right)\right], \\
& \psi_{x x}-\psi_{t t}-\sin \psi=\alpha_{2} \psi_{t}-\eta_{2}, \\
& \psi_{x}(0, t)=0, \quad \psi_{x}\left(l_{2}, t\right)=R_{12}^{-1}\left[V_{0}(t)-\psi_{t}\left(l_{2}, t\right)\right], \\
& V_{0, t t}+2 \alpha V_{0, t}+\left(\omega_{0}^{2}+a^{2}\right) V_{0} \\
& \quad=\left(R_{12} C\right)^{-1}\left[\phi_{t t}\left(l_{1}, t\right)+\psi_{t t}\left(l_{2}, t\right)\right],
\end{aligned}
$$

where $R_{12}$ is the normalized value of the resistor between each LJJ and the $L C$ tank. Solving Eqs. (5) the method of integration in time was changed, since the transient time, as expected, in this system was found to be extremely long. Typically we had to integrate the system to 4000-5000 time units to observe steady-state motion. The method used here was an explicit second-order finite-difference method, which is to our knowledge the fastest for this kind of system. The speed of integration is at least five times faster for the second-order finitedifference method than for the fourth-order Runge Kutta method used to solve the Eqs. (4) and (1). For the parameters $l_{1}=l_{2}=4, \quad \eta_{1}=\eta_{2}=0.4, \quad \alpha_{1}=0.05, \quad \alpha_{2}=0.055$, $R_{12}=7, a=\frac{1}{75}, \omega_{0}=0.74$ the motion of the system is shown in Fig. 3 as a time evolution of the tank and soliton boundary voltages [Fig. 3(a)] and as the tank phase space [Fig. 3(b)]. The three coupled oscillators are found to be phase locked at a frequency $\omega_{s}=0.743$, which is just above the tank resonance frequency. Clearly we see that the soliton of the low-loss LJJ hits the tank more out of phase than the soliton of the high-loss LJJ $\left(\left|\theta_{1}\right|>\left|\theta_{2}\right|\right)$. In that way the difference in excess energy of the two LJJ is compensated to produce the phase locked state observed. Synchronization was observed for many parameter choices. Thus we expect phase locking between the oscillators to be present for a substantial range of difference in parameter space of the two junctions. Using the map approach it was possible to demonstrate ${ }^{3}$ phase locking for a somewhat similar situation in which the junction lengths were $1 \%$ different, and a large range of bias values resulted in locked states. For a difference of
$10 \%$ in losses Fig. 4 shows the result for parameter choice: $l_{1}=l_{2}=4, \alpha_{1}=0.05, \alpha_{2}=0.055, R_{12}=7, a=\frac{1}{75}$, $\omega_{0}=0.75$. The simulation started at bias values $\eta=\eta_{1}=\eta_{2}=0.3$, for which value the oscillators did not lock. Increasing the bias by steps of 0.01 , transient times of 5000 units and averages over 1000 units the oscillators were found to be phase locked in the range $\eta=0.46 \rightarrow \eta=0.72$. At $\eta=0.73$ the soliton of the lowloss LJJ disappeared. The very large locking range is visible in Fig. 4(a) where the two $I V$ curves of the LJJ's are shown. Clearly we find that the two curves are separated for low bias values. Near the lower end of the locking range we find strong interaction between the oscillators. It is important to note that without the coupling (the $L C$ tank) there would be no intersection of the $I V$ curves of the two $\mathrm{LJJ}$.

In Fig. 4(b) we show the maximum value of the tank voltage. In the locking range we see the expected resonance curve with maximum at the bias value for which $\omega_{s}=\omega_{0}$ [see Fig. 4(a)]. Just below the locking range we find relatively large maxima of tank voltages. These are caused by the temporary in-phase motion of the two LJJ that takes place in the beating process of the unlocked states. When the beat frequency is low (near the locking
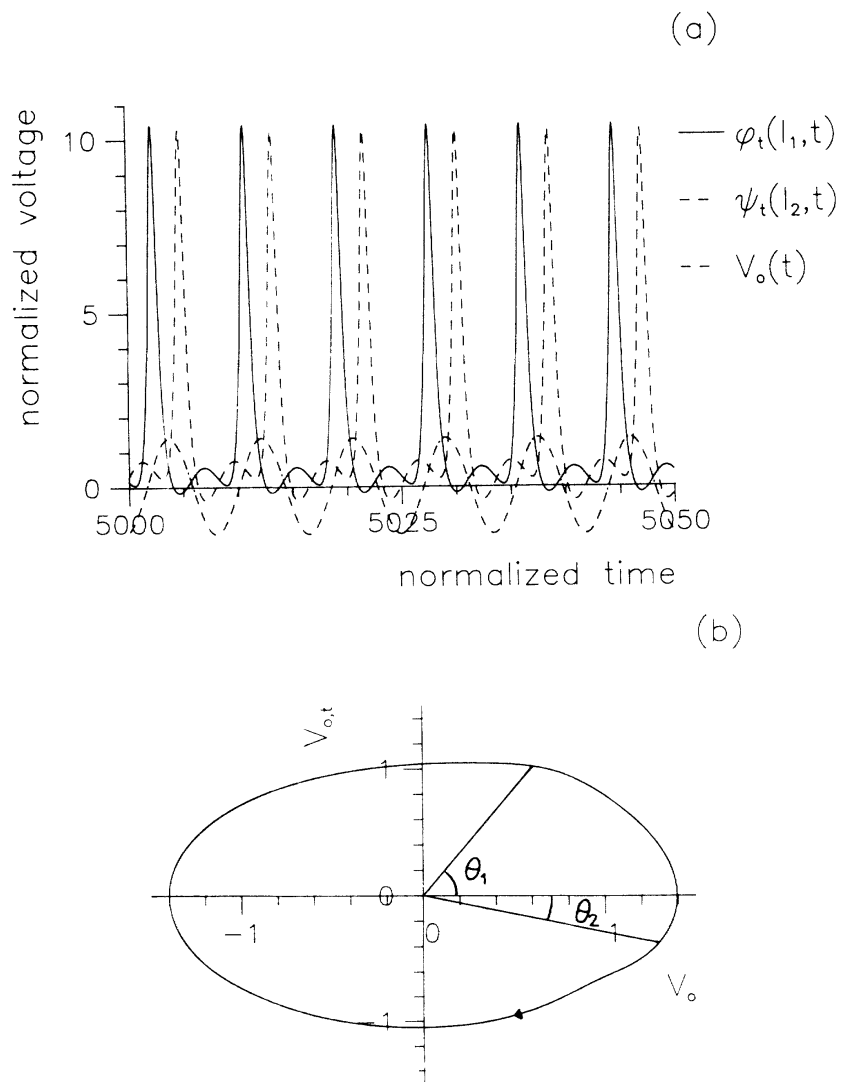

FIG. 3. The time evolution of the system of two different LJJ's coupled to a tank. The parameter set is $\eta_{1}=\eta_{2}=0.4$, $l_{1}=l_{2}=4, \alpha_{1}=0.05, \alpha_{2}=0.055, R_{12}=7, a=\frac{1}{75}, \omega_{0}=0.74$. (a) The time evolution of the tank voltage and the tank boundary voltages of the two LJJ's. (b) The tank voltage phase space in the same time interval as (a). 
(a)

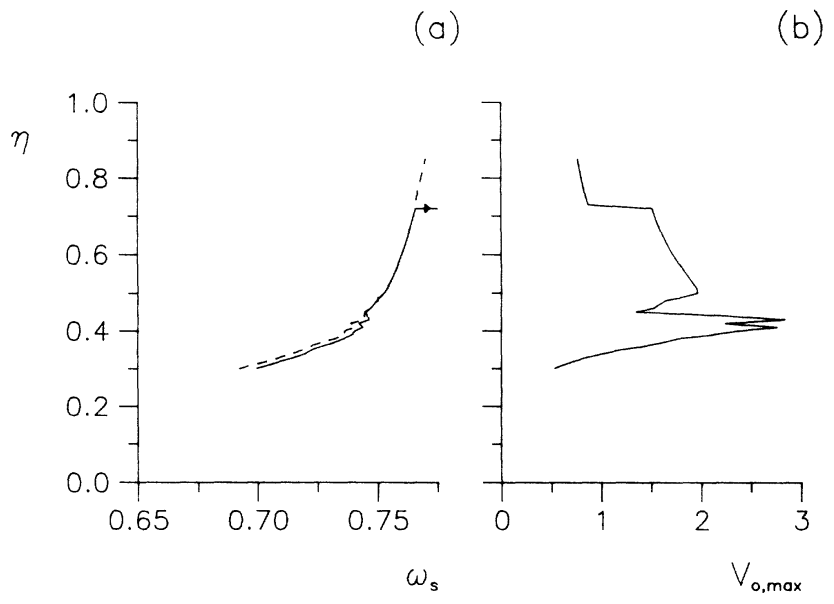

(c)

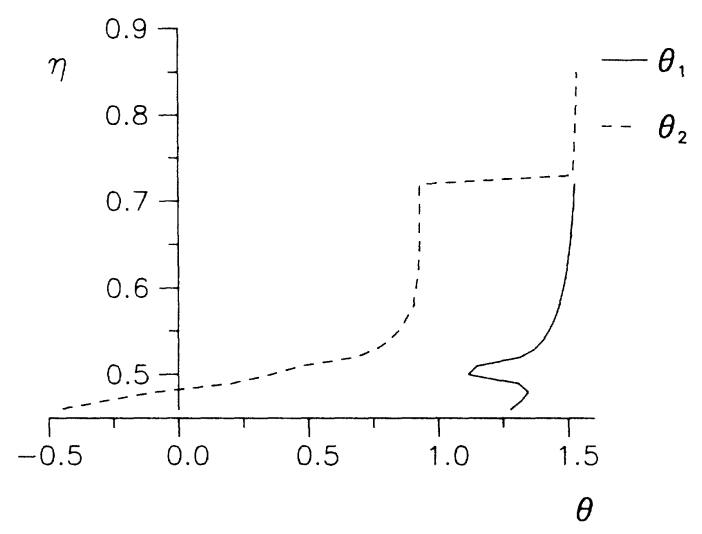

FIG. 4. Variation with the common bias value $\left(\eta=\eta_{1}=\eta_{2}\right)$. Parameters are the following: $l_{1}=l_{2}=4, \alpha_{1}=0.05, \alpha_{2}=0.055$, $R_{12}=7, a=\frac{1}{75}, \omega_{0}=0.75$. (a) Average of the soliton frequencies $\omega_{s}$. The vertical dotted line indicates the tank resonance frequency $\omega_{0}$. In normalized units the average voltages are $2 \omega_{s}$. (b) Maximum tank voltage. (c) Characteristic phase angles.

range) the tank voltage is pumped up temporarily. Above the locking range no soliton is present in the lowloss LJJ. The tank is then driven by the soliton motion in the high-loss LJJ only. A discontinuity is seen in Fig. 4(b) when the action on the tank shifts from both soliton motions to one soliton motion only.

We finally show in Fig. 4(c) the characteristic phases of the two solitons in the locked states. As expected we find that $\left|\theta_{1}\right|>\left|\theta_{2}\right|$ in the locked states, in order to allow the soliton of the low-loss LJJ to transfer a larger amount of excess energy than the other soliton. We note that the phase $\theta_{1}$ is pulled down in some sort of resonance dip when $\theta_{2} \approx 0$, i.e., when the soliton of the high-loss LJJ is in phase with the tank. Above the locking range the phase $\theta_{2}$ switches from $\theta_{2} \approx 1$ to $\theta_{2} \approx \pi / 2$ in order to make the soliton motion of only one LJJ pull the tank.

In conclusion, we note that most features from the simple map approach are confirmed in the present PDE simulations. Detailed information of the time evolution is found using a full PDE simulation of the system, but it is clear that for scanning the parameter space, the map approach is much more convenient. For longer junctions the agreement will probably be even more detailed since the collision time will then be a small fraction of the soliton transit time (or the cavity resonance period $2 \pi / \omega_{0}$ ).

The ultimate objective of the present study is to provide a workable model for experimental devices of the type reported in Ref. 2 . In this connection, we note that Monaco ${ }^{9}$ has recently performed detailed measurements on a 20 -junction, series-biased, $X$-band array similar to that described by Pagano et $a .^{2}$ In this work he was able to bias the array in such a way that a variable number, from one to ten, of individual junctions participated in the locked state. Salient results of his measurements are the following: (a) the locking range in bias current varies linearly with the number of participating junctions; (b) the power (above the noise level) of the signal emitted by the array varies with the square of the number of participating junctions. A successful model will obviously have to be able to capture these experimental phenomena.

\section{ACKNOWLEDGMENTS}

The support of the European Economic Community twinning program "Stimulated nonlinear effects in Josephson devices" [No. ST 2-0267-J-C(A)] is acknowledged. We are grateful to $\mathrm{R}$. Monaco for a prepublication discussion of his recent experimental results.
${ }^{1}$ D. G. Crete, P. Feautrier, M. Hanus, R. Monaco, and P. T. Encrenaz, in Stimulated Effects in Josephson Devices, edited by M. Russo and G. Costabile (World Scientific, Singapore, 1990), pp. 87-98.

${ }^{2}$ S. Pagano, R. Monaco, and G. Costabile, IEEE Trans. Magn. MAG-25, 1080 (1989).

${ }^{3}$ N. Grønbech-Jensen, R. D. Paramentier, and N. F. Pederesen, Phys. Lett. 142, 427 (1989).

${ }^{4}$ D. W. McLaughlin and A. C. Scott, Phys. Rev. A 18, 1652 (1978); O. A. Levring, N. F. Pedersen, and M. R. Samuelsen,
J. Appl. Phys. 54, 987 (1983)

${ }^{5}$ N. F. Pedersen, M. R. Samuelsen, and D. Welner, Phys. Rev. B 30, 4057 (1984).

${ }^{6}$ O. H. Olsen, N. F. Pedersen, M. R. Samuelsen, H. Svensmark, and D. Welner, Phys. Rev. B 33, 168 (1986).

${ }^{7}$ P. L. Christiansen and O. H. Olsen, Physica 1D, 412 (1980).

${ }^{8}$ M. Cirillo, A. R. Bishop, and P. S. Lomdahl, Phys. Rev. B 39, 4804 (1989).

${ }^{9}$ R. Monaco (private communication). 